\title{
Infrared Spectra of High Polymers. III. Polytetrafluoroethylene and Polychlorotrifluoroethylene*
}

\author{
C. Y. Liang $\dagger$ AND S. KRIMM \\ The Harrison M. Randall Laboratory of Physics, University of Michigan, Ann Arbor, Michigan
}

(Received October 27, 1955)

\begin{abstract}
The infrared spectra of polytetrafluoroethylene and polychlorotrifluoroethylene have been obtained between $4000 \mathrm{~cm}^{-1}$ and $70 \mathrm{~cm}^{-1}$. Polarization measurements on oriented samples were obtained in the region of $4000 \mathrm{~cm}^{-1}$ to $300 \mathrm{~cm}^{-1}$. It is known from $\mathrm{x}$-ray diffraction studies that both polymers have helical chain configurations. From a factor group analysis of the one-dimensional space group, selection rules and approximate vibrational patterns of the infrared active fundamentals have been derived. A calculation of the normal frequencies of an assumed planar zig-zag chain model of $\left(\mathrm{CF}_{2}\right)_{n}$ has been made, resulting in a satisfactory assignment of the bands in the infrared spectra of both polymers.
\end{abstract}

\section{INTRODUCTION}

$\mathbf{I}^{\mathrm{N}}$ the first paper of this series ${ }^{1}$ the experimental techniques used to obtain the infrared spectra of high polymers in the range of about $4000 \mathrm{~cm}^{-1}$ to $70 \mathrm{~cm}^{-1}$ were described, as well as the general theoretical methods which can be employed to interpret these spectra. In the present paper we discuss the application of these methods to the interpretation of the spectra of polytetrafluoroethylene and polychlorotrifluoroethylene.

The samples used in the present study were commercial film, and orientation was introduced by drawing. The infrared spectra of these two polymers are shown in Figs. 1 and 2, the solid and dashed curves representing the spectra obtained with radiation polarized, respectively, perpendicular and parallel to the direction of drawing, except below $350 \mathrm{~cm}^{-1}$, where the spectrum is shown with unpolarized radiation. The polarization of the intense band at $203 \mathrm{~cm}^{-1}$ in polytetrafluoroethylene was determined by utilizing the polarizing property of the grating. ${ }^{2}$ For the particular grating used (360 lines/in.), the reflected energy near $50 \mu$ contains about $75 \%$ of the component with electric vector parallel to the grooves. Thus by inserting the same oriented sample, first with the stretching direction parallel to, and then perpendicular to, the groove direction, and noting the relative absorption in both positions, it is possible to determine whether an absorption band is polarized parallel or perpendicular to the stretching direction. In this way it was found that the $203 \mathrm{~cm}^{-1}$ band is a perpendicular band $(\sigma)$, signifying that the absorption is greater when the electric vector is perpendicular to the direction of drawing than when it is parallel to this direction. For a parallel band $(\pi)$ the reverse situation is true.

The absorption spectrum of polytetrafluoroethylene in the range of $4000 \mathrm{~cm}^{-1}$ to $100 \mathrm{~cm}^{-1}$ has been reported

\footnotetext{
* Presented in part at the American Physical Society meeting, Baitimore, March, 1955.

$\dagger$ Present address: American Viscose Corporation, Marcus Hook, Pennsylvania.

${ }^{1}$ Liang, Krimm, and Sutherland, J. Chem. Phys. 25, 543 (1956).

${ }_{2}$ Peters, Zipf, and Deibel, J. Opt. Soc. Am. 43, 816 (1953), and private communication.
}

previously. ${ }^{3}$ The reflection spectrum of this polymer has been obtained ${ }^{4}$ in the range of about $1600 \mathrm{~cm}^{-1}$ to $1000 \mathrm{~cm}^{-1}$. It is in agreement with our absorption spectrum, showing the two strong bands at $1152 \mathrm{~cm}^{-1}$ and $1207 \mathrm{~cm}^{-1}$. The polarization properties of the bands at $1213,1154,641,556$, and $513 \mathrm{~cm}^{-1}$ were also obtained, ${ }^{4}$ and are in agreement with our present observations. The temperature dependence of some of the bands has been studied, ${ }^{4}$ the only variation being in a band at $630 \mathrm{~cm}^{-1}$, which is absent at $0^{\circ} \mathrm{C}$, present as a shoulder at room temperature, and equal in intensity to the $641 \mathrm{~cm}^{-1}$ band at $50^{\circ} \mathrm{C}$. (In our room temperature spectrum, the latter band occurs at $636 \mathrm{~cm}^{-1}$, and the temperature-dependent band is not observed.) No information is available on the temperature dependence of the bands below $500 \mathrm{~cm}^{-1}$.

\section{POLYTETRAFLUOROETHYLENE}

\section{Structure and Spectrum}

A detailed structure of polytetrafluoroethylene, based on x-ray diffraction studies, has recently been proposed by Bunn and Howells. ${ }^{5}$ The individual polymer chains are found to have a helical configuration and to be approximately hexagonally close-packed. The fiber-axis identity period is $16.8 \mathrm{~A}$, and corresponds to a planar zig-zag carbon chain which is twisted $180^{\circ}$ about its axis in this distance and contains 13 carbon atoms (Fig. 3, (a) and (b)). As a result, the distance between alternate carbon atoms is increased from $2.54 \mathrm{~A}$ (in the planar zig-zag) to $2.59 \mathrm{~A}$, presumably as a result of an increase in the $\mathrm{C}-\mathrm{C}-\mathrm{C}$ angle. Although a full twist of the chain occurs in $33.6 \mathrm{~A}$, the identity period is half of this because the 14 th $\mathrm{CF}_{2}$ group along the chain is directly above the first. The normal to the plane of a $\mathrm{CF}_{2}$ group makes an angle of $15-20^{\circ}$ with the axis of the helix. The polymer undergoes a first-order transition at $20^{\circ} \mathrm{C}$, accompanied by a $1 \%$ change in density, and another transition at $30^{\circ} \mathrm{C}$ in which there

\footnotetext{
${ }^{3}$ S. Krimm and G. B. B. M. Sutherland, Symposium on Molecular Structure and Spectroscopy, Columbus, Ohio, June, 1952.

${ }^{4}$ T. S. Robinson and W. C. Price, Proc. Phys. Soc. (London) B66, 969 (1953).

${ }^{5}$ C. W. Bunn and E. R. Howells, Nature 174, 549 (1954).
} 


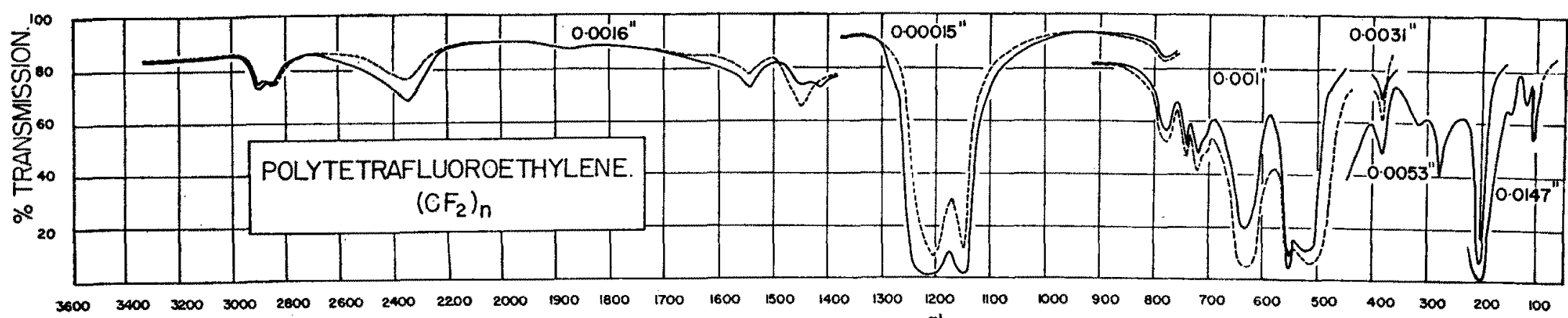
FREQUENCY in $\mathrm{cm}^{-1}$.

FIG. 1. Infrared spectrum of polytetrafluoroethylene. Numbers signify film thickness in inches. For the polarized spectrum $\left(3300-350 \mathrm{~cm}^{-1}\right):-\mathrm{electric}^{-1} \mathrm{ector}$ perpendicular to draw direction, - - - electric vector parallel to draw direction.

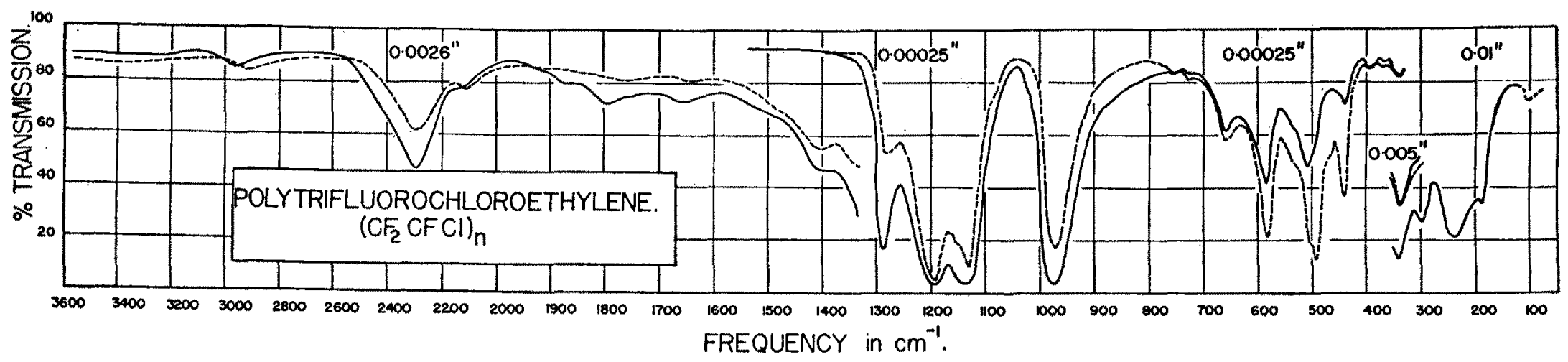

Frg. 2. Infrared spectrum of polychlorofluoroethylene. Numbers signify film thickness in inches. For the polarized spectrum $\left(3300-350 \mathrm{~cm}^{-1}\right):-$ electric vector perpendicular to draw direction, - - - electric vector parallel to draw direction. 
TABLE I. Symmetry species, irreducible representations, number of normal modes, and selection rules (A-active, F-forbidden) for polytetrafuoroethylene under the group $\mathrm{C}(14 \pi / 13)$.

\begin{tabular}{|c|c|c|c|c|c|c|c|c|c|c|c|c|c|c|}
\hline & $\mathrm{E}$ & $\mathrm{C}^{1}$ & $\mathrm{C}^{2}$ & $\ldots$ & $\mathrm{C} n$ & $\ldots$ & $\mathrm{C}^{12}$ & $n_{i}$ & $T$ & $T^{\prime}$ & $R^{\prime}$ & $n i^{\prime}$ & $I R$ & Raman \\
\hline$A$ & 1 & 1 & 1 & $\cdots$ & 1 & $\cdots$ & 1 & 9 & $T_{\pi}$ & 2 & 3 & 3 & A & A \\
\hline 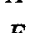 & 1 & $\epsilon^{1}$ & $\epsilon^{2}$ & $\ldots$ & $\epsilon^{n}$ & $\ldots$ & $\epsilon^{12}$ & 9) & $\pi$ & 2 & 3 & 3 & A & $\hat{\mathbf{A}}$ \\
\hline$E_{l}$ & 1 & $\epsilon^{-1}$ & $\overrightarrow{E^{-2}}$ & $\cdots$ & $e^{-n}$ & $\cdots$ & $\varepsilon^{-12}$ & $9\}$ & $T_{\sigma}$ & 2 & 3 & 3 & $\mathrm{~A}$ & A \\
\hline & 1 & $\epsilon^{2}$ & $\epsilon^{4}$ & $\cdots$ & $e^{2 n}$ & $\cdots$ & $\epsilon^{24}$ & 9 & 0 & 3 & 3 & 3 & $\mathrm{~F}$ & $\mathrm{~A}$ \\
\hline$E_{1}$ & $\bar{l}$ & $\epsilon^{-2}$ & $\epsilon^{-4}$ & $\ldots$ & $\epsilon^{-2 n}$ & $\ldots$ & $\epsilon^{-24}$ & 9 & 0 & 3 & 3 & 3 & $\mathrm{~F}$ & A \\
\hline . & . & . & • & & $\cdot$ & & . & - & . & . & . & • & . & $\cdot$ \\
\hline . & . & . & , & & . & & - & . & . & . & . & • & . & . \\
\hline . & . & . & · & & • & & . & . & . & . & . & . & . & . \\
\hline$p$ & 1 & $\epsilon^{6}$ & $\epsilon^{12}$ & $\ldots$ & $\epsilon^{6 n}$ & $\cdots$ & $\epsilon^{72}$ & 9 & 0 & 3 & 3 & 3 & $F$ & F \\
\hline$E_{6}$ & 1 & $\epsilon^{-6}$ & $e^{-12}$ & $\cdots$ & $e^{-6 n}$ & $\ldots$ & $e^{-72}$ & 9 & 0 & 3 & 3 & 3 & $\mathrm{~F}$ & $\mathrm{~F}$ \\
\hline
\end{tabular}

$\varepsilon=\operatorname{expir}(14 \pi / 13)]$

is an additional smaller change in density. From the changes in the $x$-ray diffraction patterns at these temperatures, it is concluded that the translational order between chains in the crystal is disturbed but the internal molecular configuration of any given chain is not altered. It will therefore be satisfactory to assume that the interaction between neighboring chains is small, and that the spectrum may be interpreted in terms of the structure of a single chain, i.e., in terms of a onedimensional lattice.

Although the symmetry of a single chain may be described in terms of a $13_{2}$ or $13_{11}$ screw axis, the following equivalent description is more convenient. Consider, as in the above structure, an infinitely long helical chain of $\mathrm{CF}_{2}$ groups containing 13 groups in the repeating unit. No assumption is made at this stage about the orientation of a $\mathrm{CF}_{2}$ group with respect to the axis of the helix. The symmetry operation required to bring any $\mathrm{CF}_{2}$ group into coincidence with an adjacent $\mathrm{CF}_{2}$ group consists of a rotation of $(\pi+\pi / 13)$ about the axis of the helix followed by a translation along the axis of $1 / 13$ of the identity period of $16.8 \mathrm{~A}$. We denote this operation by $C^{1}$. (A spectroscopically equivalent structure, the mirror-image of the above, is obtained if the angle of rotation is $(\pi-\pi / 13)$ in the same sense as above.) The third $\mathrm{CF}_{2}$ group along the helix is obtained from the first chosen $\mathrm{CF}_{2}$ group by the symmetry operation consisting of a rotation of $2(\pi+\pi / 13)$ about the axis followed by a translation along the axis of $2 / 13$ of the identity period. This symmetry operation is denoted by $C^{2}$, and signifies that the symmetry operation $C^{1}$ is performed twice in succession. By similar symmetry operations, the succeeding $\mathrm{CF}_{2}$ groups are obtained from the first (see Fig. 3c). The operation $C^{13}$ is equivalent to a translation equal to the identity period, and is denoted by $E$, the identity operation. The sequence of symmetry elements $E, C^{1}, C^{2}, C^{3}, \cdots C^{12}$ forms a cyclic group which is a factor group of the onedimensional space group corresponding to an infinite helical chain. We may denote this factor group by $C(14 \pi / 13)$.
From the factor group analysis ${ }^{6}$ are obtained the symmetry species, irreducible representations, total number of normal modes, $n_{i}$, under the $i$ th irreducible representation, and the selection rules for fundamentals in the infrared and Raman spectra. These are given in Table I, where in addition the subdivision of the normal modes into pure translations $(T)$ and into translatory $\left(T^{\prime}\right)$, rotatory $\left(R^{\prime}\right)$, and internal $\left(n_{i}^{\prime}\right)$ types of motion is shown. The infrared active modes are those under species $A$ and $E_{1}$, and will exhibit parallel $(\pi)$ and perpendicular $(\sigma)$ dichroism, respectively. The Raman active modes are those under species $A, E_{1}$, and $E_{2}$.

In the foregoing we assumed a general orientation of the $\mathrm{CF}_{2}$ groups. If we also require, as seems to be implied from the Bunn and Howells structure, that the axis of each $\mathrm{CF}_{2}$ group is perpendicular to and intersects the axis of the helix, additional symmetry elements are then present. These are the twofold axes coinciding with the axes of the $\mathrm{CF}_{2}$ groups. When these symmetry elements are added to the group we obtain a factor group of the one-dimensional space group which can be designated as $D(14 \pi / 13)$. The species, characters,
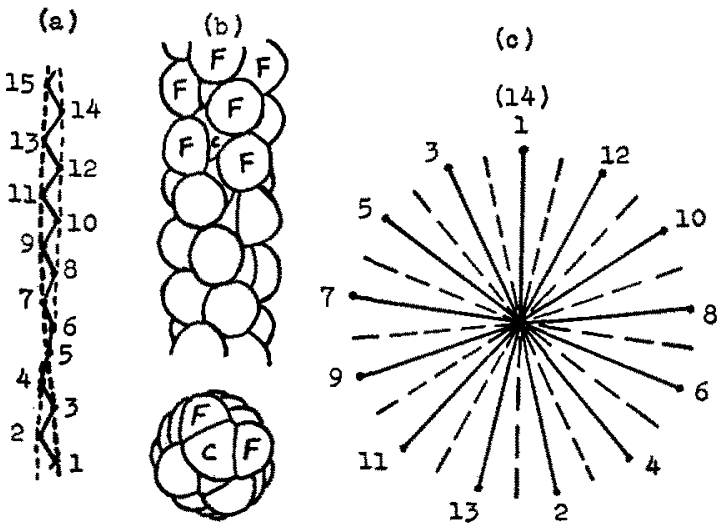

FIG. 3. Structure of polytetrafluoroethylene. (a) Twisted carbon zig-zag chain. (b) Side and end views of the molecule. (c) Symmetry relations between $\mathrm{CF}_{2}$ groups in the polymer.

- S. Bhagavantam and T. Venkatarayudu, "Theory of groups and its application to physical problems," Andhra University, Waltair, India (1951). 
TABLE II. Symmetry species, characters, number of normal modes, and selection rules (A-active, F-forbidden) for polytetrafiuoroethylene under the group $D(14 \pi / 13) .^{\mathrm{a}}$

\begin{tabular}{|c|c|c|c|c|c|c|c|c|c|c|}
\hline & $\mathrm{E}$ & $2 \mathrm{C}^{1}$ & $2 \mathrm{C}^{2}$ & $\ldots$ & $2 \mathrm{C}^{6}$ & $13 C_{2}$ & $n_{i}$ & $T$ & $I R$ & Raman \\
\hline$A_{1}$ & 1 & 1 & 1 & $\ldots$ & 1 & 1 & 4 & & $\mathrm{~F}$ & A \\
\hline$A_{2}$ & 1 & 1 & 1 & $\ldots$ & 1 & -1 & 5 & $T_{\pi}$ & A & $\mathrm{F}$ \\
\hline$E_{1}$ & 2 & $2 \cos \omega^{\mathrm{b}}$ & $2 \cos 2 \omega$ & $\ldots$ & $2 \cos 6 \omega$ & 0 & 18 & $T_{\sigma}^{4}$ & A & $\mathrm{A}$ \\
\hline$E_{2}$ & 2 & $2 \cos 2 \omega$ & $2 \cos 4 \omega$ & $\cdots$ & $2 \cos 12 \omega$ & 0 & 18 & & $\mathrm{~F}$ & A \\
\hline : & $\dot{.}$ & . & : & & $\dot{.}$ & . & $\dot{.}$ & & $\mathbf{F}$ & $\mathrm{F}$ \\
\hline$E_{6}$ & 2 & $2 \cos 6 \omega$ & $2 \cos 12 \omega$ & & $2 \cos 36 \omega$ & 0 & 18 & & $\mathrm{~F}$ & $\mathrm{~F}$ \\
\hline
\end{tabular}

a We give here the conjugate classes rather than the individual symmetry elements. The elements of $C^{j}$ and $C^{j}$ belong to a conjugate class if $i=13-j$. $\mathrm{b} \omega=14 \pi / 13$.

number of normal modes, and selection rules under this factor group are given in Table II. It will be noted that the nondegenerate modes are now divided into $4 A_{1}$ and $5 A_{2}$, only the latter being infrared active.

A detailed analysis of combinations and overtones can only be made from the finite space group. ${ }^{7}$ However, the rules governing those combinations and overtones which arise only from the infrared active fundamentals can be obtained from the factor group. The polarization properties of such combinations and overtones, as determined from the factor group analysis, are given in Table III for the two symmetry groups. The polarization properties of the fundamentals are also included. There will in addition be the possibility of obtaining infrared active combination bands from active and inactive fundamentals. These have not been examined in great detail here, since the positions of the infrared inactive fundamental frequencies are at present unknown.

\section{Normal Modes of Vibration}

The approximate vibrational patterns for the active fundamentals can be deduced from the normal modes of a $\mathrm{CF}_{2}$ group and the symmetry of the molecule. The symmetry species correspond physically to modes of vibration of the helix in which there are various possible phase differences between given $\mathrm{CF}_{2}$ motions in neighboring groups. If we denote this phase difference by $\theta$, then $\theta=(14 \pi / 13) r$ with $r=0$ for the nondegenerate modes, and $r= \pm 1$ and \pm 2 for the degenerate modes $E_{1}$ and $E_{2}$, respectively. The vibrational pattern for a species $A$ mode is therefore one in which the motions in all $\mathrm{CF}_{2}$ groups are in phase with one another, and that for a species $E_{1}$ mode is one in which motions in neighboring $\mathrm{CF}_{2}$ groups differ in phase by $\pm 14 \pi / 13$. In this way $^{8}$ it is possible to build up approximate normal modes of the chain from those of the $\mathrm{CF}_{2}$ group, and these are shown in Fig. 4 for a repeat unit of the helix. This vibrational pattern must be reproduced identically in all of the other repeat units of the helix if the mode is to be active in the infrared or Raman spectrum. On the left are shown the nine basic modes of a $\mathrm{CF}_{2}$ group, $\nu_{1}, \nu_{2}$, and $\nu_{3}$ corresponding to translations, $\nu_{6}, \nu_{8}$, and $\nu_{9}$ to rotations, and $\nu_{4}, \nu_{5}$, and $\nu_{7}$ to the internal vibrations. The infrared active vibrations under the $A$ species arising from these $\mathrm{CF}_{2}$ modes are shown in the second column. ' (It should be noted that under the dihedral group $D(14 \pi / 13)$ the nondegenerate modes $\nu_{3}, \nu_{4}, \nu_{5}$, and $\nu_{9}$ belong to the infrared inactive species $A_{1}$, while the remaining nondegenerate modes belong to species $A_{2}$ and are infrared active.) For $\nu_{1}$ to $\nu_{5}$ the end view of the chain is shown; for $\nu_{6}$ to $\nu_{9}$ the side view. The dots in the end view modes represent the equilibrium positions of the $\mathrm{CF}_{2}$ groups. In the side view modes the fluorine atoms above and below the plane of the paper are represented by open and closed circles, respectively. For the vibrational patterns $\nu_{4}$ through $\nu_{7}$, each arrow denotes the trend of the dipole moment change of a $\mathrm{CF}_{2}$ group; for the other modes, the arrows and the + and - signs represent the displacement of atoms or groups of atoms from their equilibrium positions.

The approximate vibrational patterns of the degenerate modes are shown in the last two columns of Fig. 4. Only one component of each degenerate pair is

TABLE III. Polarization properties of infrared active fundamentals, overtones, and combinations under the factor groups $C(14 \pi / 13)$ and $D(14 \pi / 13)$ in the infrared.

\begin{tabular}{|c|c|c|c|c|}
\hline & \multicolumn{2}{|c|}{$C(14 \pi / 13)$} & \multicolumn{2}{|c|}{$D(14 \pi / 13)$} \\
\hline & Species & Polarization & Species & Polarization \\
\hline \multirow{4}{*}{$\begin{array}{l}\text { Fundamentals } \\
\text { Overtones and combinations }\end{array}$} & $A$ & $\pi$ & $A_{2}$ & $\pi$ \\
\hline & $E_{1}$ & $\sigma$ & $E_{1}$ & $\sigma$ \\
\hline & $A \cdot A=A$ & $\pi \cdot \pi=\pi$ & $A_{2} \cdot A_{2}=A_{1}$ & $\pi \cdot \pi$ : inactive \\
\hline & $\begin{array}{l}A \cdot E_{1}=E_{1} \\
\left(E_{1}\right)^{2}=A+E_{2} \\
E_{1} \cdot E_{1}=A+E_{2}\end{array}$ & $\begin{array}{l}\pi \cdot \sigma=\sigma \\
(\sigma)^{2}=\pi \\
\sigma \cdot \sigma=\pi\end{array}$ & $\begin{array}{l}A_{2} \cdot E_{1}=E_{1} \\
\left(E_{1}\right)^{2}=A_{1}+E_{2} \\
E_{1} \cdot E_{1}=A_{1}+A_{2}+E_{2}\end{array}$ & $\begin{array}{l}\pi \cdot \sigma=\sigma \\
(\sigma)^{2}: \text { inactive } \\
\sigma \cdot \sigma=\pi\end{array}$ \\
\hline
\end{tabular}

${ }^{7}$ H. Winston and R. S. Halford, J. Chem. Phys. 17, 607 (1949).

${ }^{8} \mathrm{G}$. Herzberg, Infrared and Raman Spectra (D. Van Nostrand Company, Inc., New York, 1945). 
shown, the motions being projected along a direction parallel or perpendicular to the axis of a $\mathrm{CF}_{2}$ group. The other component of the pair is obtained by a rotation of the pattern of vibrational amplitudes (considered independently of the atomic positions) about the helix axis and a translation along the axis giving a new pattern which is orthogonal to the original one. (In the case of species $E_{1}$ the angle of rotation is $90^{\circ}$ and the translation is one half of the identity period.) The method for deriving such degenerate pairs is shown in detail for the modes $\nu_{1}, \nu_{2}$, and $\nu_{3}$ under species $E_{1}$ in Fig. 5. This makes clear the manner in which the nongenuine degenerate vibrations, viz., the translations, arise. It will be noted that in general the normal mode is a combination of the two components of the degenerate pair, thus giving elliptical or circular vibrational modes. From Figs. 4 and 5 , it can be seen that the modes have the following approximate character: $\nu_{1}$-translatory skeletal motion parallel to the helix axis (the $A$ species being a pure translation); $\nu_{2}$-rotatory skeletal motion about the helix axis, (the $A$ species being a pure rotation); $\nu_{3}$-translatory skeletal motion perpendicular to the helix axis (the $E_{1}$ species being a pure translation) $; \nu_{4}$-symmetric $\mathrm{CF}_{2}$ stretching; $\nu_{5}-\mathrm{CF}_{2}$ bending; $\nu_{6}-\mathrm{CF}_{2}$ rocking; $\nu_{7}$-asymmetric $\mathrm{CF}_{2}$ stretching ; $\nu_{8}-\mathrm{CF}_{2}$ wagging; $\nu_{9}-\mathrm{CF}_{2}$ twisting.

Approximate intensities for the vibrational modes of the helix can now be predicted on the basis of the foregoing discussion and the structure of the molecule. It is reasonable to expect that the vibrations associated with the $\mathrm{CF}_{2}$ stretching, both symmetrical and asymmetrical, bending, wagging, and rocking modes should give rise to a large dipole moment change in a single $\mathrm{CF}_{2}$ group. The addition of these moments in a given helix mode will then determine the net change in dipole moment, and therefore the strength of the absorption band. It will thus be seen that the strong bands should be associated with $\nu_{4}\left(E_{1}\right), \nu_{7}\left(E_{1}\right), \nu_{5}\left(E_{1}\right)$, $\nu_{8}(A)$ (or $\nu_{8}\left(A_{2}\right)$ for the dihedral group), and $\nu_{6}\left(E_{1}\right)$. Because the plane of a $\mathrm{CF}_{2}$ group is inclined at a small angle to the axis of the helix, we would expect medium or weak bands corresponding to $\nu_{6}\left(A\right.$ or $\left.A_{2}\right), \nu_{7}\left(A\right.$ or $\left.A_{2}\right)$, and $\nu_{8}\left(E_{1}\right)$ to appear as well in the spectrum. The remaining fundamentals would most probably give rise to weak bands, as would combinations and overtones. It should be noted that the nondegenerate modes $\nu_{3}, \nu_{4}$, $\nu_{5}$, and $\nu_{9}$ are infrared active for the group $C(14 \pi / 13)$. A spectroscopic decision as to which group the polytetrafluoroethylene molecule belongs would therefore have to depend upon the presence or absence of these bands and an analysis of the combinations and overtones.

\section{Calculation of Normal Frequencies}

From the predicted polarization, the expected intensity, and knowledge of the approximate regions in which the characteristic frequencies are found, the
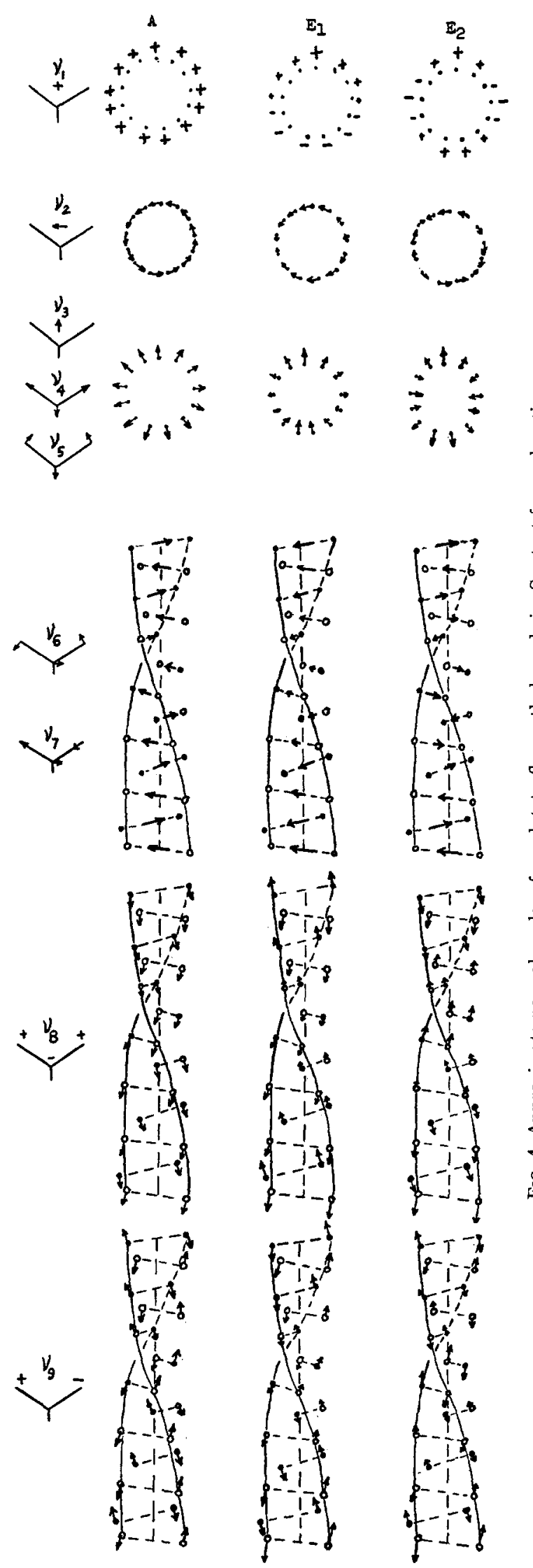

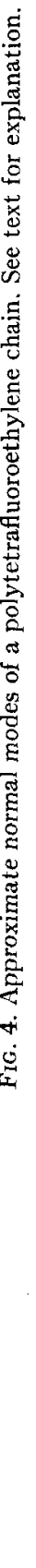



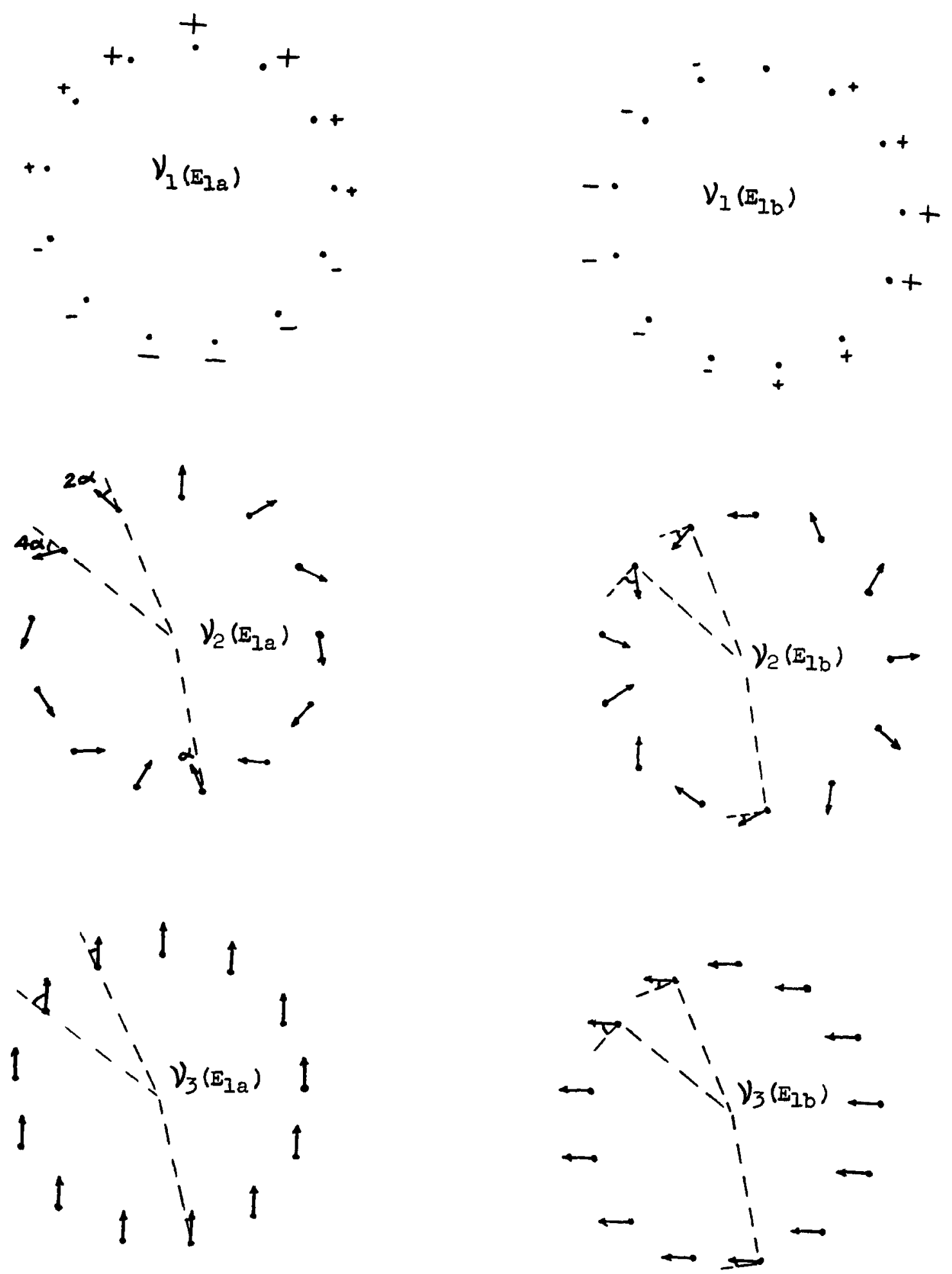

FIG. 5. Degenerate pairs of $\nu_{1}\left(E_{1}\right), \nu_{2}\left(E_{1}\right)$, and $\nu_{\hat{v}}\left(E_{1}\right)$.

four strong $\sigma$ bands in the spectrum of polytetrafluoroethylene can be assigned unambiguously. We will defer the discussion of the assignment of these bands until the next section. The assignment of the two intense $\pi$ bands as well as the remaining weaker bands, however, does not follow clearly from the above considerations alone. In order to achieve a more complete assignment, the normal frequencies of a hypothetical planar zig-zag 
chain model of $\left(\mathrm{CF}_{2}\right)_{n}$ have been calculated. The reason for choosing such a model is that the calculations are much easier than for the actual helical structure, and it may reasonably be expected that these frequencies will be close to the frequencies of the helix, particularly since the distortion from a planar zig-zag is relatively small.

The method of calculation follows that given by Kellner $^{9}$ for an infinite planar zig-zag chain of $\mathrm{CH}_{2}$ groups, the same formulas being used in the present case. The angles in the chain are taken as tetrahedral, the equilibrium bond lengths as $1.54 \mathrm{~A}$ for $\mathrm{C}-\mathrm{C}$ and $1.35 \mathrm{~A}$ for $\mathrm{C}-\mathrm{F}$ bonds. The force constants were chosen such that they were close to values obtained from previous work on fluorinated hydrocarbons ${ }^{10}$ and gave calculated frequencies which were near the observed strong bands in the expected regions. The values of the force constants which were used are: the stretching force constants $f_{\mathrm{CF}}=5.37$ and $f_{\mathrm{CC}}=3.46$; the bending force constants $\phi_{\mathrm{FCF}}=0.990, \phi_{\mathrm{CCF}}=0.623, \phi_{\mathrm{CCC}}=0.289$; and the twisting force constant $\tau=0.026$, all in units of $10^{5}$ dynes $/ \mathrm{cm}$. The results of this calculation are shown in Table IV, the symmetry class designation being in terms of the factor group of the one-dimensional space group, $D_{2 h}$, rather than the point group $C_{2 h}$ used by Kellner. ${ }^{1,11}$ The normal modes of the helical molecule which would correspond to the modes of the planar model are also indicated, the designation being that of Fig. 4. Although for the planar zig-zag chain the exclusion rule holds, so that only some of these frequencies are infrared active, this restriction would not maintain for the helical structure, and we would expect all of these frequencies to be permitted in general in the infrared spectrum. On the basis of the above calculation of normal frequencies, and the previous discussion, we can immediately assign the expected five strong bands in the spectrum. In Table $\mathrm{V}$ are listed the frequency, relative intensity, and polarization of the observed bands, together with the assignments to be discussed below.

Following from the above calculations we see that the $\sigma$ band at $1207 \mathrm{~cm}^{-1}$ is assigned to $\nu_{7}\left(E_{1}\right)$, a $\mathrm{CF}_{2}$ asymmetrical stretching frequency; the $\sigma$ band at $1152 \mathrm{~cm}^{-1}$ is assigned to $\nu_{4}\left(E_{1}\right)$, a $\mathrm{CF}_{2}$ symmetrical stretching frequency; and the $\pi$ band at $636 \mathrm{~cm}^{-1}$ is assigned to $\nu_{8}\left(A_{2}\right)$, a $\mathrm{CF}_{2}$ wagging frequency. (We will discuss shortly our reasons for preferring $D(14 \pi / 13)$ to $C(14 \pi / 13)$.) The $\sigma$ band at $553 \mathrm{~cm}^{-1}$ is assigned to $\nu_{5}\left(E_{1}\right)$, a $\mathrm{CF}_{2}$ deformation mode, and the $\sigma$ band at $203 \mathrm{~cm}^{-1}$ is assigned to $\nu_{6}\left(E_{1}\right)$, a $\mathrm{CF}_{2}$ rocking vibration. It will be noted that the frequencies of these bands agree quite well with the calculated values.

These five bands are expected to be strong, and are

${ }^{9}$ L. Kellner, Proc. Phys. Soc. (London) A64, 521 (1951).

${ }^{10}$ E. L. Pace, J. Chem. Phys. 16, 74 (1948); H. H. Claasen, ibid. 18, 543 (1950); Decker, Meister, and Cleveland, ibid. 19, 784 (1951).

11 M. C. Tobin, J. Chem. Phys. 23, 891 (1955).
TABLE IV. Frequencies of hypothetical planar model of $\left(\mathrm{CF}_{2}\right)_{n}$.

\begin{tabular}{|c|c|c|c|c|}
\hline \multirow{2}{*}{$\begin{array}{c}\text { Symmetry } \\
\text { class } \\
\left(D_{2 h}\right) \\
\end{array}$} & \multirow{2}{*}{$\begin{array}{c}\text { Planar model } \\
\text { Approximate character } \\
\text { of vibration }\end{array}$} & \multirow{2}{*}{$\begin{array}{l}\text { Calculated } \\
\text { frequency } \\
\mathrm{cm}^{-1}\end{array}$} & \multicolumn{2}{|c|}{$\begin{array}{l}\text { Corresponding helical } \\
\text { chain mode }\end{array}$} \\
\hline & & & $C(14 \pi / 13)$ & $D(14 \pi / 13)$ \\
\hline$B_{\text {su }}$ & translation, parallel to & $\mathbf{0}$ & $\nu_{1}$ & $A z$ \\
\hline$B_{a} a$ & $\begin{array}{l}\text { chain axs } \\
\text { rotation, about chain axis }\end{array}$ & 0 & $\boldsymbol{\nu}_{2}$ & $A_{2}$ \\
\hline$A_{\mathcal{G}}$ & chain stretching & 650 & $v_{3}$ & $A_{1}$ \\
\hline $\begin{array}{l}A_{g} \\
A_{g}\end{array}$ & $\begin{array}{l}\mathrm{CF}_{2} \mathrm{Sym}_{\text {. }} \text { stretching } \\
\mathrm{CF}_{2} \text { bending }\end{array}$ & $\begin{array}{r}1460 \\
375\end{array}$ & $\nu_{\nu_{5}} A$ & $\begin{array}{l}A_{2} \\
A_{1}\end{array}$ \\
\hline$B_{30}$ & $\mathrm{CF}_{2}$ rocking & & $\nu_{0}$ & \\
\hline$B_{30}$ & $\mathrm{CF}_{2}$ asym, stretching & 1595 & $\eta 7$ & $A_{2}$ \\
\hline$B_{3 u}$ & CF 2 wagging & 630 & $\nu 8$ & $A_{2}$ \\
\hline${ }_{B}^{A} u_{1}$ & $\mathrm{CF}_{2}$ twisting & 310 & $\nu_{9}$ & $A_{1}$ \\
\hline $\begin{array}{l}B_{19} \\
B_{14}\end{array}$ & $\begin{array}{l}\text { chain stretching } \\
\text { translation, } \perp \text { chain axis }\end{array}$ & $\begin{array}{r}1300 \\
0\end{array}$ & $\nu_{1}$ & \\
\hline$B_{2 u}$ & translation, $\perp$ chain axis & 0 & $\nu_{2}$ & \\
\hline$B_{2 u}$ & $\mathrm{CF}_{2}$ sym. stretching & 1149 & & \\
\hline & $\mathrm{CF}_{2}$ bending & 562 & $\mu_{k} E_{1}$ & $E_{1}$ \\
\hline & $\mathrm{CF}_{2}$ rocl & 204 & $\nu_{6}$ & \\
\hline$B_{34}$ & $\begin{array}{l}\mathrm{CF}_{2} \text { asym. stretching } \\
\mathrm{CF}_{2} \text { wagging }\end{array}$ & $\begin{array}{r}1230 \\
270\end{array}$ & $\begin{array}{l}\nu_{7} \\
\nu_{8}\end{array}$ & \\
\hline$B_{2 g}$ & $\mathrm{CF}_{2}$ twisting & 323 & עo & \\
\hline
\end{tabular}

found to be so in the spectrum. The remaining strong band found in the spectrum, the $\pi$ band at $520 \mathrm{~cm}^{-1}$, is not as easy to account for. On the basis of the calculation for the planar model, we expect a $\mathrm{CF}_{2}$ rocking frequency, $\left(B_{3 \theta}\right)$ near $540 \mathrm{~cm}^{-1}$. This would correspond to the helix mode $\nu_{6}\left(A_{2}\right)$ of $\pi$ polarization, but as we have seen earlier it is not expected to be as intense a band as the above five bands. Its strength could be accounted for, however, by a Fermi resonance with the fundamental mode $\nu_{8}\left(A_{2}\right)$, thus giving rise to the two strong $\pi$ bands at $636 \mathrm{~cm}^{-1}$ and $520 \mathrm{~cm}^{-1}$. Although we will designate the former as a $\mathrm{CF}_{2}$ wagging mode and the latter as a $\mathrm{CF}_{2}$ rocking mode, it must be understood that if Fermi resonance occurs these designations are only very approximate, since both bands would then be mixtures of both of these modes.

The other two weaker bands expected in the spectrum are associated with $\nu_{8}\left(E_{1}\right)$ and $\nu_{7}\left(A_{2}\right)$. The former is

TABLE V. Interpretation of the infrared spectrum of polytetrafluoroethylene.

\begin{tabular}{|c|c|c|c|c|}
\hline $\begin{array}{l}\text { Frequency } \\
\mathrm{cm}^{-1}\end{array}$ & R.I. & Polarization & Assignment & $\begin{array}{l}\text { Calculated } \\
\text { frequency }\end{array}$ \\
\hline 102 & $w$ & $\cdots$ & & \\
\hline 124 & $w$ & $\cdots$ & & \\
\hline 149 & $\mathrm{w}$ & $\cdots$ & & \\
\hline 203 & $\mathbf{s}$ & $\sigma$ & $\nu_{6}\left(E_{1}\right)$ & 204 \\
\hline 277 & $\mathrm{~m}$ & $\cdots$ & $\nu_{8}\left(E_{1}\right)$ & 270 \\
\hline 321 & $w$ & $\cdots$ & $\nu_{9}\left(E_{1}\right)$ & 323 \\
\hline 384 & $\mathrm{~m}$ & $\pi$ & & \\
\hline 520 & $\mathbf{s}$ & $\pi$ & $\nu_{6}\left(A_{2}\right)$ & 540 \\
\hline 553 & $\mathrm{~s}$ & $\sigma$ & $\nu_{5}\left(E_{1}\right)$ & 562 \\
\hline 620 & shoulder & $\cdots$ & & \\
\hline 636 & $\mathbf{s}$ & $\pi$ & $\nu_{8}\left(A_{2}\right)$ & 630 \\
\hline 720 & $\mathrm{~m}$ & $\pi$ & & \\
\hline 742 & $\mathrm{~m}$ & $\pi$ & & \\
\hline 780 & $\mathrm{~m}$ & $\pi$ & & \\
\hline 1152 & vs & $\sigma$ & $\nu_{4}\left(E_{1}\right)$ & 1149 \\
\hline 1207 & vs & $\sigma$ & $\nu_{i}\left(E_{1}\right)$ & 1230 \\
\hline 1410 & vw & $\sigma$ & & \\
\hline 1450 & $\mathbf{w}$ & $\pi$ & $\nu_{7}\left(A_{2}\right)$ & 1595 \\
\hline 1545 & vw & $\sigma$ & & \\
\hline 2350 & $\mathbf{w}$ & $\sigma$ & & \\
\hline 2850 & vw & $\pi(?)$ & & \\
\hline 2905 & vw & $\sigma$ & & \\
\hline
\end{tabular}


approximately a $\mathrm{CF}_{2}$ wagging mode, and is found at $270 \mathrm{~cm}^{-1}$ in the planar model. We therefore assign the band at $277 \mathrm{~cm}^{-1}$ to $\nu_{8}\left(E_{1}\right)$. The other mode, $\nu_{7}\left(A_{2}\right)$, a $\mathrm{CF}_{2}$ asymmetrical stretching mode, is found at 1595 $\mathrm{cm}^{-1}$ in the planar model, and is expected to have $\pi$ polarization in the helix. The only band found in the spectrum which can be assigned to this mode is the $\pi$ band at $1450 \mathrm{~cm}^{-1}$. Although the difference between calculated and observed frequencies is fairly large $(9.1 \%)$, the assignment may be considered satisfactory if the only other possibility, viz., the assignment of $\nu_{4}(A)$ to the $1450 \mathrm{~cm}^{-1}$ band (corresponding to the $1460 \mathrm{~cm}^{-1}$ frequency of the planar model), can be eliminated. We therefore turn now to the consideration of the occurrence of $\nu_{3}(A), \nu_{4}(A)$, and $\nu_{5}(A)$ in the spectrum, which would imply, as we have seen earlier, that the axis of the $\mathrm{CF}_{2}$ group is other than perpendicular to the axis of the helix.

If assignments for $\nu_{3}(A), \nu_{4}(A)$, and $\nu_{5}(A)$ are attempted on the basis of a comparison with the frequencies calculated for the hypothetical planar model, the following correlations can be made which seem to be reasonable:

$\begin{array}{ccc}\text { Assignment } & \begin{array}{c}\text { Observed } \\ \text { frequency }\end{array} & \begin{array}{c}\text { Calculated } \\ \text { frequency }\end{array} \\ \nu_{3}(\mathrm{~A}) & 720 & 650 \\ \nu_{4}(\mathrm{~A}) & 1450 & 1460 \\ \nu_{5}(\mathrm{~A}) & 384 & 375\end{array}$

The discrepancy is large only for $\nu_{3}(A)$, viz., about $10.8 \%$. There are serious difficulties, however, with this assignment. First, if we accept the group $C(14 \pi / 13)$, it can be seen from Table III that overtones of the infrared fundamentals are permitted in the infrared, and we should expect to find overtones of the two very strong $\mathrm{CF}_{2}$ stretching modes at $1152 \mathrm{~cm}^{-1}$ and 1207 $\mathrm{cm}^{-1}$. Not only do we not find bands which fit numerically, but the only band in the appropriate region, the weak band at $2350 \mathrm{~cm}^{-1}$, has the wrong polarization for such an overtone. On the other hand, under the group $D(14 \pi / 13)$ such overtones are forbidden in the infrared, and this would agree with the observed results. Second, there is evidence $\mathrm{e}^{12}$ that the band at $720 \mathrm{~cm}^{-1}$ is to be associated with the amorphous part of the specimen: it is found to become weaker as the sample becomes more crystalline. This would eliminate the possibility of assigning $\nu_{3}(A)$. Finally, the acceptance of $C(14 \pi / 13)$ has the most likely implication that the axis of each $\mathrm{CF}_{2}$ group is inclined at an angle other than $90^{\circ}$ to the axis of the helix. It would be quite difficult to justify this conclusion on structural grounds. For the above reasons, we are therefore led to favor the dihedral group for polytetrafluoroethylene.

It is thus pertinent to inquire into other possible assignments for the observed $\pi$ bands at $384 \mathrm{~cm}^{-1}$, between 700 and $800 \mathrm{~cm}^{-1}$, and at $1450 \mathrm{~cm}^{-1}$. We have already discussed the assignment of the $1450 \mathrm{~cm}^{-1}$ band

${ }^{12}$ C. F. Hammer (private communication). to $\nu_{7}\left(A_{2}\right)$, and the probable assignment of the bands between 700 and $800 \mathrm{~cm}^{-1}$ to the amorphous chains. It is quite possible that the $384 \mathrm{~cm}^{-1}$ band is also due to the molecules in the amorphous state. A more careful examination of samples with varying crystallinity would be desirable in order to check this point.

The identification of the remaining fundamentals with the other observed bands is less certain. By comparison with the $\mathrm{CF}_{2}$ twisting mode in the planar model, viz., $B_{20}$ at $323 \mathrm{~cm}^{-1}$, it is probable that the observed band at $321 \mathrm{~cm}^{-1}$ is associated with $\nu_{9}\left(E_{1}\right)$, the $\mathrm{CF}_{2}$ twisting mode. Lack of polarization data for this band prevents a definite assignment. Of the chain stretching frequencies, we have seen that $\nu_{3}\left(A_{1}\right)$ is prohibited under the dihedral group. The stretching mode $B_{1 \rho}$ of the planar model, corresponding approximately to $\nu_{1}\left(E_{1}\right)$ of the helix, is predicted at about $1300 \mathrm{~cm}^{-1}$. There is no band in the spectrum assignable to $\nu_{1}\left(E_{1}\right)$, and it is in fact possible that this frequency is overlapped with the $\mathrm{CF}_{2}$ stretching bands in the $1200 \mathrm{~cm}^{-1}$ region. The $\nu_{2}\left(E_{1}\right)$ chain stretching mode is likewise not identifiable in the spectrum. The low-lying bands at $102 \mathrm{~cm}^{-1}, 124 \mathrm{~cm}^{-1}$, and $149 \mathrm{~cm}^{-1}$ have not been assigned, but they may possibly be lattice vibrations or difference bands. The change in intensity of the shoulder at $620 \mathrm{~cm}^{-1}$ is not interpretable at present. The band may be due to crystal perturbations, and therefore reflect a change in crystal structure with temperature. On the other hand, it may arise from changes in the chain configuration. A careful determination of the temperature dependence of the remainder of the spectrum might help to clarify this point.

\section{POLYCHLOROTRIFLUOROETHYLENE}

\section{Structure and Spectrum}

The detailed structure of polychlorotrifluoroethylene has not as yet been determined. Kaufman ${ }^{13}$ has reported some $x$-ray diffraction results which indicate that the unit cell is hexagonal with $a_{0}=6.5 \mathrm{~A}$ and $c_{0}=35 \mathrm{~A}$. The long fiber axis repeat is thought to indicate that the chain configuration is a helical one, analogous to that of polytetrafluoroethylene, containing about 14 monomer units in the repeat. (The monomer unit is $-\mathrm{CF}_{2} \mathrm{CFCl}-$.) We have also obtained $\mathrm{x}$-ray diffraction patterns of oriented polychlorotrifluoroethylene. The layer-line spacings give, however, a fiber-axis repeat of $43 \mathrm{~A}$ rather than the $35 \mathrm{~A}$ repeat found by Kaufman. Very strong near-meridian reflections on the second and sixteenth layer lines are consistent with a helical chain configuration containing 16 monomer units in the $43 \mathrm{~A}$ identity period. It is therefore possible that helical chain configurations of different pitch can be obtained under different conditions.

The symmetry of such a helical molecule can be

${ }^{13}$ H. S. Kaufman, J. Am. Chem. Soc. 75, 1477 (1953). 
TABLE VI. Interpretation of the infrared spectrum of polychlorotrifuoroethylene.

\begin{tabular}{|c|c|c|c|c|}
\hline $\begin{array}{l}\text { Frequency } \\
\mathrm{cm}^{-1}\end{array}$ & $\begin{array}{l}\text { Relative } \\
\text { intensity }\end{array}$ & Polarization & Species & $\begin{array}{l}\text { Approximate } \\
\text { character of vibration }\end{array}$ \\
\hline 102 & $\mathrm{w}$ & & & \\
\hline 185 & $\mathrm{w}$ & & $E_{\mathrm{l}}$ & CClF rocking \\
\hline 235 & $\mathrm{~m}$ & & $E_{1}$ & $\mathrm{CF}_{2}$ rocking \\
\hline 297 & $\mathrm{w}$ & & $E_{1}$ & $\mathrm{CF}_{2}$ wagging (?) \\
\hline 335 & $\mathrm{~m}$ & unpol. & $E_{1}$ & CCIF bending \\
\hline 365 & $\mathrm{w}$ & $\cdots$ & & \\
\hline 390 & $\mathrm{w}$ & $\pi ?$ & & \\
\hline 438 & $\mathrm{~s}$ & $\pi$ & $A$ & CCIF wagging \\
\hline 490 & $\mathrm{~s}$ & $\pi$ & $A$ & $\mathrm{CF}_{2}$ rocking \\
\hline $\begin{array}{r}506 \\
(535)\end{array}$ & $s$ & $a ?$ & $E_{1}$ & $\mathrm{CF}_{2}$ bending \\
\hline 580 & $\mathbf{s}$ & $\pi$ & $A$ & $\mathrm{CF}_{2}$ wagging \\
\hline 658 & $\mathrm{~m}$ & $\pi$ & & \\
\hline 723 & $\mathrm{w}$ & $\pi$ & & \\
\hline 755 & w & $\sigma ?$ & & \\
\hline 972 & vs & $\sigma$ & $E_{1}$ & $\mathrm{CCl}$ stretching \\
\hline 1130 & vs & $\sigma$ & $E_{1}$ & $\mathrm{CF}_{2}$ stretching \\
\hline$(1147)$ & & $\sigma$ & & \\
\hline 1194 & vs & $\sigma$ & $E_{1}$ & $\mathrm{CF}_{2}$ stretching \\
\hline 1285 & $\mathrm{~s}$ & $\sigma$ & $E_{1}$ & CF stretching \\
\hline 1400 & vw & $\cdots$ & & \\
\hline 1660 & $\mathrm{vw}$ & $\sigma$ & & \\
\hline 1800 & vw & $\sigma$ & & \\
\hline 1870 & $\mathrm{vW}$ & $\sigma$ & & \\
\hline 2110 & $\mathrm{vw}$ & $\pi ?$ & & \\
\hline 2294 & $\mathrm{~m}$ & $\sigma$ & & \\
\hline
\end{tabular}

described, as in the case of polytetrafluoroethylene, in terms of a cyclic group which is a factor group of the one-dimensional space group of the infinite helix. Although the exact number of monomer units in the repeat of the helix may not be known, it can be shown ${ }^{14}$ that in any case the same number of active fundamentals will be obtained, in this case 18 under species $A$, 18 under species $E_{1}$, and 18 under species $E_{2}$. The modes under $A$ and $E_{1}$ are infrared active while the modes under $A, E_{1}$, and $E_{2}$ are Raman active. The infrared active bands under species $A$ should exhibit $\pi$ dichroism while those under species $E_{1}$ should show $\sigma$ dichroism. The phase difference, $\theta$, between identical motions in adjacent monomer units will of course depend upon the number of monomer units in the repeat.

It is not possible to identify all of the 36 predicted infrared active fundamentals. A satisfactory assignment can be made, however, as a result of the analogies between the spectra of polytetrafluoroethylene and poly-

${ }^{14}$ P. W. Higgs, Proc. Roy. Soc. (London) A220, 472 (1953).

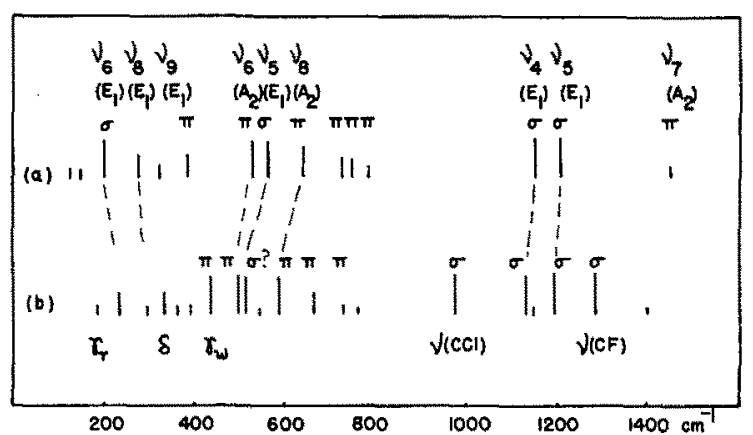

Fro. 6. Correlations between the spectra of (a) polytetrafluoroethylene and (b) polychlorotrifluoroethylene.

chlorotrifluoroethylene. In Fig. 6 we show the correlations which seem to be possible between the two spectra. These correlations serve to more or less unambiguously identify the $\mathrm{CF}_{2}$ frequencies in polychlorotrifluoroethylene. Most of the remaining bands in the spectrum are undoubtedly due to vibrations associated with the $\mathrm{CFCl}$ group, and these can be assigned without too much difficulty on the basis of the frequency region and the polarization properties of the band. The resulting interpretation of the spectrum is given in Table VI. Since the motions of the atoms in the chain are probably quite mixed, the designation of the character of the vibration is only meant to be suggestive of the approximate physical nature of the vibration.

We may note in connection with the above assignments that they are in good agreement with the assignments for the monomer. ${ }^{15}$ It is also of interest that the $\mathrm{CF}_{2}$ frequencies in this polymer can be correlated with those in polytetrafluoroethylene. In view of the established helical structure of the latter polymer, this correlation provides strong spectroscopic evidence for a helical structure for polychlorotrifluoroethylene.

\section{ACKNOWLEDGMENTS}

The authors wish to express their indebtedness to Professor G. B. B. M. Sutherland for helpful discussions and suggestions during the course of this work. Part of the work was supported by grants to the authors from E. I. duPont de Nemours and Company, for which we wish to express our appreciation.

\footnotetext{
${ }^{15}$ Mann, Acquista, and Plyler, J. Chem. Phys. 21, 1949 (1953).
} 